\title{
Descrição dos acidentes domésticos ocorridos na infância
}

Description of domestic accidents during childhood

\author{
Ludmila Mourão Xavier-Gomes* \\ Renata Mendes Rocha** \\ Thiago Luis de Andrade-Barbosa*** \\ Carla Silvana de Oliveira e Silva****
}

\begin{abstract}
Resumo
Os acidentes domésticos infantis são importante fonte de preocupação, pois são causas crescentes de mortalidade e invalidez no Brasil. O objetivo deste estudo foi identificar e descrever os acidentes domiciliares na infância na área de abrangência de uma Estratégia de Agente Comunitário de Saúde (EACS) de Montes Claros-MG, Brasil. Trata-se de um estudo descritivo, retrospectivo, quantitativo realizado por meio de questionário estruturado com pais ou responsáveis de crianças de zero a 12 anos no ambiente domiciliar. Participaram do estudo 50 pais ou responsáveis pelas crianças. Foram registrados 104 acidentes domésticos. Do total de crianças, 19 (38,0\%) eram do sexo masculino e 31 (62\%) do sexo feminino. De acordo com a idade, seis acidentes $(12,0 \%)$ ocorreram com crianças de três meses a oito meses, 15 acidentes $(30,0 \%)$ ocorreram com crianças de oito meses a menor de quatro anos, 11 acidentes $(22,0 \%)$ ocorreram com crianças de quatro a menor de oito anos e 18 acidentes (36,0\%) ocorreram com crianças de oito anos a 12 anos. O estudo mostra que a presença dos pais não evita que os acidentes ocorram, já que em $97(93,3 \%)$ acidentes as crianças estavam acompanhadas pelos pais. Os tipos de acidentes que ocorreram foram: choque elétrico; colisão; cortes; escorregão; intoxicação; obstrução de vias aéreas; queda; queimadura. Houve predomínio das quedas. Concluiu-se que as crianças são muito vulneráveis aos acidentes e que o ambiente doméstico pode trazer riscos ou segurança.
\end{abstract}

Palavras-chave: Acidentes. Criança. Acidentes Domésticos.

\section{Abstract}

The childhood home accidents are an important source of concern, as they are growing causes of mortality and disability in Brazil. The aim of this study was to identify and describe childhood home accidents of a Health Community Agent Strategy (EACS) in Montes Claros, Minas Gerais, Brazil. This is a descriptive, retrospective, quantitative study accomplished through a structured questionnaire applied with parents or guardians of children aged between zero and 12 years in the home environment. The study included fifty parents or guardians. One hundred and four household accidents were recorded. Nineteen children (38.0\%) were male and $31(62.0 \%)$ were female. Regarding age, six accidents $(12.0 \%)$ occurred among children aged from three to eight months, 15 accidents (30.0\%) involved children from eight months to less than four-years, 11 accidents $(22.0 \%)$ occurred in children from four to less than eight years and 18 accidents $(36.0 \%)$ occurred with children from eight to 12 years. The study shows that the parents' presence does not prevent accidents from occurring, since in 97 (93.3\%) accidents the children were accompanied by the parents. The types of accidents registered were electric shock, impact, cuts, slips, poisoning, airway obstruction, fall and burn. Among these, falls were the most common. It was concluded that children are very vulnerable to accidents and that the home environment may involve risks or safety.

Keywords: Accidents. Child. Accidents, Home.

\footnotetext{
* Enfermeira. Mestre em Ciências da Saúde. Doutoranda em Ciências da Saúde pela Universidade Federal de Minas Gerais. Líder do Grupo de Pesquisa em Enfermagem FASA das Faculdades Santo Agostinho de Montes Claros, Montes Claros-MG, Brasil. E-mail: ludyxavier@ yahoo.com.br

** Enfermeira. Graduada pelas Faculdades Santo Agostinho de Montes Claros, Montes Claros-MG, Brasil.

*** Enfermeiro. Mestre em Ciências da Saúde. Doutorando em Ciências da Saúde pela Universidade Estadual de Montes Claros, Montes Claros-MG, Brasil.

**** Enfermeira. Doutoranda em Ciências pela Universidade Federal de São Paulo. Coordenadora pedagógica do curso de Graduação em Enfermagem das Faculdades Santo Agostinho de Montes Claros. Docente da Universidade Estadual de Montes Claros, Montes Claros-MG, Brasil. Os autores declaram não haver conflitos de interesse.
} 


\section{INTRODUCุÃO}

Distante da violência das ruas, a casa oferece riscos que causam a morte de cinco mil crianças por ano no Brasil. De acordo com dados do Ministério da Saúde, lesões não intencionais representam a principal causa de morte na faixa etária de zero a 14 anos de idade no País ${ }^{1}$.

Observa-se uma tendência da produção científica sobre acidentes e violência apresentada em encontros de iniciação científica. Porém, apesar de os acadêmicos estarem investigando sobre a temática, a produção científica ainda é insuficiente, considerando a magnitude do problema².

Os principais tipos de acidentes domésticos encontrados são quedas; contusões; cortes; queimaduras; escoriações; esmagamentos; mordeduras; e perfurações. No geral, nota-se que o ambiente doméstico está sendo atingido cada vez mais pelo desenvolvimento tecnológico, tornando-se, em algumas situações, altamente perigoso para as crianças, devido à sua característica de ser um centro de atividades diárias para a família ${ }^{3}$.

O grupo infantil é o mais vulnerável aos desastres e acidentes no estrato populacional. Quanto mais jovem e imatura for a criança, menor sua percepção de risco e maior sua vulnerabilidade e dependência de terceiros em termos de segurança contra acidentes e desastres. A vulnerabilidade da criança aos acidentes é variável em função do nível de coordenação de seu sistema nervoso, aptidão motora, senso de percepção de risco e da instintiva proteção a ela dispensada pela mãe e demais familiares ${ }^{4}$.

Os acidentes domésticos são frequentes porque os pais nem sempre conhecem as limitações de cada fase da vida dos filhos, além de não terem o hábito de pensar nos perigos dentro de casa. É comum que os adultos esperem da criança uma percepção de risco, que ela desenvolve só a partir dos sete anos. Até os quatro anos, ela é curiosa, mistura o real e o fictício e imita os adultos. A partir dos quatro anos, são movidos pelo desafio. Isso sem contar que cai com mais facilidade porque a cabeça é desproporcional ao corpo, fazendo com que o centro de gravidade seja no peito e não no umbigo. A partir daí, a criança é menos ingênua, mas passa a ser movida pelo desafio. São características que os pais precisam conhecer e levar em conta para maior segurança dos filhos ${ }^{1}$.

Além disso, merecem questionamentos sobre a atitude paternalista que não considera a criança como um ser em desenvolvimento, que evolui continuamente à medida que habilidades se aprimoram e novas capacidades são adquiridas ${ }^{5}$.

No Brasil, os acidentes domésticos infantis são causas crescentes de mortalidade e invalidez e importante fonte de preocupação, constituindo-se no grupo predominante de causas de morte a partir de um ano de idade ${ }^{6}$.

Em média, dois terços de todos os acidentes com crianças ocorrem dentro de casa, e a maioria poderia ter sido evitada. É oportuno salientar que não é de se surpreender que a maior parte dos acidentes ocorra no domicílio, haja vista o grande período que as crianças pequenas permanecem nele ou em seus limites. Isso exige que os responsáveis pelas crianças tenham conhecimento dos riscos do ambiente, tomando medidas para evitá-los, pois, ao mesmo tempo que o domicílio pode propiciar a ocorrência desses agravos, ele pode funcionar também como um meio facilitador para ações preventivas e educativas, neutralizando a existência de tal risco $^{7,8}$.

Na literatura científica, verifica-se uma imensa desproporção entre a importância do tema em relação à morbimortalidade de crianças e jovens e o escasso número de estudos a ele relacionados. Descreve, ainda, que injúrias físicas constituem um enorme problema de saúde pública entre crianças e jovens. No entanto, invisíveis aos olhos da maioria, uma vez que, apesar dos maus prognósticos, continuam sendo menosprezados ${ }^{9}$.

O Ministério da Saúde define acidente como o evento não intencional e evitável, que causa lesões físicas e/ou emocionais no âmbito doméstico ou nos outros ambientes sociais. Os acidentes podem ocorrer em maior ou menor grau, perfeitamente previsíveis, e preveníveis por intermédio da orientação familiar, de alterações físicas do espaço domiciliar e da elaboração e ou cumprimento de leis específicas ${ }^{10}$. 
Nessa perspectiva, o objetivo geral deste estudo foi identificar e descrever os acidentes domiciliares na infância na área de abrangência de uma Estratégia de Agente Comunitário de Saúde (EACS) de Montes Claros-MG.

\section{MÉTODO}

Trata-se de estudo descritivo, retrospectivo, quantitativo, desenvolvido em Montes Claros, município de médio porte, localizado na região norte do estado de Minas Gerais, Brasil. Apresenta população aproximada de 350 mil habitantes, contando com 52 equipes completas da Estratégia Saúde da Família e 11 equipes da Estratégia de Agentes Comunitários de Saúde na zona urbana.

Foram considerados elegíveis para o estudo todos os pais ou responsáveis das crianças de zero a 12 anos da área de abrangência da Estratégia de Agentes Comunitários de Saúde (EACS) Nossa Senhora Aparecida, situada em Montes Claros-MG, Brasil. Os critérios de exclusão da família foram: não encontrar os pais ou responsáveis da criança no domicílio em até três tentativas; a família ter mudado da região durante o período estudado; e a família ter se cadastrado após o intervalo de tempo estabelecido para esse estudo.

O instrumento de coleta de dados constituiu-se em um questionário estruturado para identificar os tipos de acidentes domiciliares na infância, traçar o perfil dos entrevistados e identificar a frequência dos acidentes domiciliares. No período da coleta de dados, a pesquisadora primeiramente identificou os endereços e os nomes das crianças de zero a 12 anos junto ao EACS. Posteriormente, visitou o domicílio de cada criança. Ressalta-se que houve a realização de um estudo piloto, previamente à coleta de dados com objetivo de adequar o formulário estruturado e a estratégia da entrevista para a coleta de dados.

Para a caracterização dos participantes, utilizaram-se as seguintes variáveis: sexo; idade; raça; a criança frequenta a escola; a criança mora com quem; responsável pela criança na maior parte do tempo; a criança já foi aciden- tada; classe socioeconômica; tipo de acidente; quem acompanhava a criança no momento do acidente. Os dados obtidos foram tabulados no PASW Statistics 18.0, e a análise foi realizada por meio da estatística descritiva com a utilização de frequências, porcentagens, média e desvio padrão.

A classificação socioeconômica foi realizada com base no "Critério de Classificação Econômica Brasil", que é um sistema que avalia o poder de compra de grupos de consumidores, e exclui a pretensão de classificar a população em termos de "classes sociais", dividindo o mercado exclusivamente em classes econômicas ${ }^{11}$.

Os preceitos éticos foram respeitados. Para a participação na pesquisa, o Termo de Consentimento Livre e Esclarecido foi assinado pelos pais das crianças. O projeto foi aprovado pelo Comitê de Ética em Pesquisa da Unimontes, com Parecer Consubstanciado n. 1860/2010.

\section{RESULTADOS}

Participaram do estudo 50 pais ou responsáveis de crianças de 0 a 12 anos, das quais 41 $(82,0 \%)$ sofreram acidentes, com predomínio do sexo feminino: $31(62,0 \%)$. As características das crianças são apresentadas na Tabela 1 .

Em relação à faixa etária, seis acidentes $(12,0 \%)$ ocorreram com crianças de três meses a oito meses, 15 acidentes (30,0\%), com crianças de oito meses a menor de quatro anos, 11 acidentes $(22,0 \%)$, com crianças de quatro a menor de oito anos e 18 acidentes (36,0\%), com crianças de oito anos a 12 anos.

Quanto à classe socioeconômica à qual pertencem as crianças, verificou-se que 26 pertenciam à classe $C(52,0 \%)$ e 24 , à classe D (42,0\%). De acordo com o Critério de Classificação Econômica Brasil ${ }^{11}$, a classe $C$ tem renda média de $R \$ 927,00$ reais, e a classe $D$, de R\$ 424,00 reais. Quanto à frequência escolar, $29(58,0 \%)$ das crianças frequentam escola, e $21(42,0 \%)$ não frequentam escola. Observou-se que $41(82,0 \%)$ crianças moram com os pais (mãe e pai) e nove crianças (18,0\%) moram apenas com a mãe. 
Tabela 1. Características das crianças de zero a 12 anos do EACS Nossa Senhora das Graças de Montes Claros-MG. Brasil, 2010

\begin{tabular}{|c|c|c|}
\hline Variável & $\mathbf{N}$ & $\%$ \\
\hline \multicolumn{3}{|l|}{ Sexo } \\
\hline Masculino & 19 & 38,0 \\
\hline Feminino & 31 & 62,0 \\
\hline \multicolumn{3}{|l|}{ Idade } \\
\hline De 3 meses a menor de 8 meses & 6 & 12,0 \\
\hline De 8 meses a menor de 4 anos & 15 & 30,0 \\
\hline De 4 anos a menor de 8 anos & 11 & 22,0 \\
\hline De 8 anos a 12 anos & 18 & 36,0 \\
\hline \multicolumn{3}{|l|}{ Raça } \\
\hline Branca & 9 & 18,0 \\
\hline Negra & 4 & 8,0 \\
\hline Parda & 37 & 74,0 \\
\hline \multicolumn{3}{|l|}{ Classe socioeconômica (pais) } \\
\hline $\mathrm{C}$ & 26 & 52,0 \\
\hline $\mathrm{D}$ & 24 & 48,0 \\
\hline \multicolumn{3}{|l|}{ Criança frequenta a escola? } \\
\hline Sim & 29 & 58,0 \\
\hline Não & 21 & 42,0 \\
\hline \multicolumn{3}{|l|}{ A criança mora com quem? } \\
\hline Pais & 41 & 82,0 \\
\hline Mãe & 9 & 18,0 \\
\hline \multicolumn{3}{|c|}{$\begin{array}{l}\text { Quem é responsável pela criança na maior } \\
\text { parte do tempo? }\end{array}$} \\
\hline Pai & 2 & 4,0 \\
\hline Mãe & 44 & 88,0 \\
\hline Tio(a) & 1 & 2,0 \\
\hline Avó(ô) & 2 & 4,0 \\
\hline Outros & 1 & 2,0 \\
\hline
\end{tabular}

Verificou-se que, entre as crianças pesquisadas, 41 sofreram algum tipo de acidente, sendo 104 acidentes no total. 15 crianças sofreram apenas um acidente; dez crianças sofreram acidentes duas vezes; cinco crianças se acidentaram três vezes; cinco crianças foram acidentadas cinco vezes. A descrição da quantidade de acidentes encontra-se na Tabela 2.

Tabela 2. Quantidade de acidentes por criança do EACS Nossa Senhora das Graças de Montes Claros-MG. Brasil, 2010

\begin{tabular}{lcc}
\hline Quantidade de Acidentes por criança & \multicolumn{2}{c}{ Crianças acidentadas } \\
\hline & $\mathbf{n}$ & $\%$ \\
Um & 15 & 30,0 \\
Dois & 10 & 20,0 \\
Três & 5 & 10,0 \\
Quatro & 4 & 8,0 \\
Cinco & 5 & 10,0 \\
Seis & 1 & 2,0 \\
Sete & 1 & 2,0 \\
\hline
\end{tabular}

A Tabela 3 mostra que houve predomínio de acidentes, com 47 casos, na faixa etária de oito anos a 12 anos, seguida das faixas etárias de oito meses a menor de quatro anos, com 37 casos, de quatro anos a menor de oito anos, com 14 acidentes, e de três meses a menor de oito meses, com sete acidentes.

Tabela 3. Crianças vítimas de acidentes, segundo idade e tipo de acidente. EACS Nossa Senhora das Graças de Montes Claros-MG. Brasil, 2010

\begin{tabular}{lccccc}
\hline \multicolumn{5}{c}{ IDADE CATEGORIZADA } \\
\hline VARIÁvel & $\begin{array}{c}\text { De } \mathbf{3} \text { a menor de } \\
\mathbf{8} \text { meses }\end{array}$ & $\begin{array}{c}\text { De } \mathbf{8} \text { meses a } \\
\text { menor de } \mathbf{4} \text { anos }\end{array}$ & $\begin{array}{c}\text { De } \mathbf{4} \text { a menor } \\
\text { de } \mathbf{8} \text { anos }\end{array}$ & $\begin{array}{c}\text { De 8 anos a 12 } \\
\text { anos }\end{array}$ & Total \\
\hline $\begin{array}{l}\text { Tipos de Acidentes } \\
\text { Queda }\end{array}$ & 4 & & & & \\
Choque elétrico & 1 & 25 & 9 & 28 & 66 \\
Corte & - & 1 & 1 & 2 & 6 \\
Queimadura & - & 2 & - & 4 & 5 \\
Colisão & - & - & - & 4 & 6 \\
Escorregão & 1 & 5 & 1 & 2 & 4 \\
Obstrução de vias aéreas & 1 & 1 & - & 3 & 9 \\
Intoxicação & - & 1 & - & 2 & 3 \\
Total & $\mathbf{7}$ & $\mathbf{3 7}$ & $\mathbf{1 3}$ & $\mathbf{4 7}$ & $\mathbf{1 0 4}$ \\
\hline
\end{tabular}

A Tabela 3 ainda permite verificar a predominância das quedas, com 66 casos, representando 63,5\% dos acidentes, seguidas dos escorregões $(8,6 \%)$, das queimaduras $(5,7 \%)$, dos choques elétricos $(5,7 \%)$, dos cortes $(4,8 \%)$, da obstrução de vias aéreas $(4,8 \%)$, das colisões $(3,8 \%)$ e das intoxicações $(2,9 \%)$. 
Tabela 4. Crianças vítimas de acidentes, segundo quem acompanhava no momento do acidente e tipo de acidente do EACS Nossa Senhora das Graças de Montes Claros-MG. Brasil, 2010

\begin{tabular}{l|cccccc}
\hline \multicolumn{7}{c}{$\begin{array}{c}\text { Quem } \\
\text { Acompanhava }\end{array}$} \\
\hline \multirow{2}{*}{ Tipo de Acidente } & \multicolumn{7}{c}{ Pais } & & Outros & \multicolumn{1}{c}{ Sozinho } \\
\cline { 2 - 7 } Queda & $\mathbf{n}$ & $\%$ & $\mathbf{n}$ & $\%$ & $\mathbf{n}$ & $\%$ \\
Choque elétrico & 59 & 56,7 & 5 & 4,8 & 2 & 1,9 \\
Corte & 6 & 5,8 & - & - & - & - \\
Queimadura & 5 & 4,8 & - & - & - & - \\
Colisão & 6 & 5,8 & - & - & - & - \\
Escorregão & 4 & 3,8 & - & - & - & - \\
Obstrução de vias aéreas & 9 & 8,6 & - & - & - & - \\
Intoxicação & 5 & 4,8 & - & - & - & - \\
\hline
\end{tabular}

A Tabela 4 mostra que em 97 acidentes $(93,2 \%)$ as crianças estavam acompanhadas pelos pais. Foi observado que a queda $(56,7 \%)$ foi o acidente mais comum, seguida do escorregão $(8,6 \%)$, do choque elétrico $(5,8 \%)$ e queimadura $(5,8 \%)$. Ressalta-se que, em todos esses acidentes, os pais estavam presentes. Em relação ao fato de a criança estar sozinha ou com outros a acompanhando, constatou-se que a queda foi o acidente mais frequente.

\section{DISCUSSÃO}

Os achados do estudo apontaram para caracterização dos acidentes domésticos envolvendo crianças, dados que permitiram a comparação com literatura. Primeiramente, foi observado que os acidentes foram mais frequentes entre o sexo feminino. Estudo realizado com infantes e pré-escolares detectou que a predominância de acidentes é do gênero masculino, com resultados diferentes deste estudo. O predomínio do gênero masculino foi explicado pelas diferenças de atividades desempenhadas por cada sexo. As brincadeiras e atividades desenvolvidas pelos meninos, em geral, são mais dinâmicas e de maior contato físico, enquanto que as meninas desenvolvem atividades mais brandas ${ }^{12}$.

No presente estudo, as faixas etárias de três meses a menor de oito meses e de oito meses a menor de quatro anos foram apontadas como as que mais se acidentaram. Na primeira faixa etária, observa-se que a criança, em relação à sua segurança, é extremamente dependente da mãe e dos demais adultos em situação de acidentes. Há um aumento da habilidade motora. Com o aumento da motilidade e a crescente atividade exploratória, os riscos ambientais se intensificam. Na segunda faixa etária (oito meses a menor de quatro anos), a capacidade motora desenvolve-se rapidamente. A criança aprende a andar, e crescem a curiosidade e o sentido de descoberta do mundo exterior. Com o aumento da motilidade e a crescente atividade exploratória, os riscos ambientais se intensificam. Passa a imitar as crianças mais velhas e os adultos e a envolver-se em brincadeiras com crianças maiores, o que justifica os riscos de acidentes nessa faixa etária ${ }^{4}$.

Foi observado que as quedas foram o acidente mais comum entre todas as faixas etárias. Um estudo sobre acidentes na infância em um pronto-socorro pediátrico identificou que o tipo de acidente mais frequente é a queda, com 46,9\% dos casos. Outro achado foi que a queda constituiu o tipo mais comum de acidente encontrado na faixa etária de menores de um ano e entre um a três anos, que se justifica pela fase de maturação motora, cognitiva e psicossocial que se encontram essas crianças, aprendendo a conhecer os seus limites e adaptando-se ao meio, achado que se assemelha ao presente estudo, no qual se verificou que o subgrupo de oito meses a menor de quatro anos foi representativo para o total de quedas. Ainda em relação às quedas, elas permanecem em níveis elevados também em crianças acima de quatro anos, evento relacionado provavelmente às atividades de lazer e esportes, próprios dessa faixa etária, destacando-se jogos, bicicleta, 
patins e outros. A queda também representou o tipo mais frequente de acidente na infância e demonstra que é na faixa etária de oito a 12 anos que mais ocorrem acidentes ${ }^{13,14,15}$.

Neste estudo, ocorreram cinco casos de obstruções das vias aéreas nas faixas etárias de três meses a menor de quatro anos e de oito anos a menor de 12 anos. Ressalta-se que as mortes por obstrução mecânica das vias aéreas ocorreram mais frequentemente no primeiro ano de vida, e são mais comuns com alimentos ou objetos pequenos. Os sintomas podem ser mínimos, especialmente no caso de pequenos corpos estranhos, e cerca da metade dos episódios sequer é testemunhada ${ }^{6}$.

De acordo com a Sociedade Brasileira de Pediatria, corpo estranho é qualquer objeto ou substância que inadvertidamente penetra o corpo ou suas cavidades. Pode ser ingerido ou colocado pela criança nas narinas e conduto auditivo, mas apresenta um risco maior quando é aspirado para o pulmão. Qualquer material pode se tornar um corpo estranho no sistema respiratório, e a maior suspeita de que o acidente ocorreu é a situação de engasgo. Isso ocorre quando a criança está comendo, ou quando está com um objeto na boca, habitualmente peças pequenas de brinquedos. No Brasil, milho, feijão e amendoim são os grãos mais comumente aspirados na faixa etária pediátrica. Por outro lado, o material mais relacionado a óbito imediato por asfixia é o sintético, como balões de borracha, estruturas esféricas, sólidas ou não, como bola de vidro e brinquedos ${ }^{16,17}$.

Neste estudo, as queimaduras representam o terceiro tipo de acidente mais frequente. Para a Sociedade Brasileira de Pediatria, as queimaduras representam a quarta causa de morte e hospitalização, por acidente, de crianças e adolescentes de até 14 anos. A maioria das queimaduras ocorre na cozinha e na presença de um adulto. Em muitos casos, o tratamento é muito doloroso, demorado e deixa marcas permanentes ${ }^{18}$.

Os casos de choques elétricos neste estudo representam o quarto tipo de acidente mais frequente. Segundo o guia "Crianças e Adolescentes Seguros", a incidência desse tipo de acidente em crianças vem aumentando gradativamente, sendo resultado da maior utilização de eletricidade nas residências sem os devidos cuidados. A gravidade do choque elétrico é ocasionada principalmente por queimaduras.
A lesão ocorre quando a energia elétrica é convertida em energia térmica. A intoxicação é a quinta causa de mortes infantis em acidentes domésticos, necessitando de outros cuidados mais específicos. Os medicamentos constituem uma das principais causas de intoxicação em qualquer idade, tendo maior destaque em crianças de até cinco anos, mas os produtos de limpeza estão em segundo lugar. A grande maioria dos casos de intoxicações ocorre dentro de casa. Praticamente todas as dependências do lar podem ter produtos químicos que apresentam algum potencial de risco ${ }^{20}$.

É importante destacar que os acidentes, em sua maioria, aconteceram em ocasiões em que os pais estavam acompanhando as crianças (93,2\%). Dados semelhantes foram encontrados por outros autores $^{18}$, que descrevem que, em 79,21\% dos casos, a mãe estava presente e apenas em $2,57 \%$ as crianças estavam sozinhas no domicílio. Destaca-se que a presença dos pais na residência não garante que as crianças encontram-se protegidas de acidentes. A presença dos pais não impede que o acidente ocorra, talvez por desconhecimento de como evitá-lo, ou, ainda, por não estar realizando a supervisão da criança ${ }^{13,14}$.

As limitações deste estudo se referem ao caráter retrospectivo das informações coletadas visto que dependiam da lembrança dos pais ou responsáveis das crianças acerca do acidente ocorrido. Outra questão é que por abordar apenas uma unidade de saúde do município de Montes Claros os dados são limitados ao público pesquisado.

\section{CONCLUSÃO}

De acordo com os resultados obtidos, verificou-se que as crianças são muito vulneráveis aos acidentes e que o ambiente doméstico pode trazer riscos ou segurança. Sugere-se que a segurança e proteção das crianças dependem dos responsáveis, visto que os acidentes são passíveis de serem previsíveis e prevenidos.

Nessa perspectiva, a redução dos acidentes pode ser alcançada mediante prevenções educativas com pais e responsáveis, assegurando informações e procedimentos necessários para proteger a criança em relação a esse problema. O profissional enfermeiro é apto a desenvolver programas para prevenção, sendo, portanto, de extrema importância para a redução de tais acidentes na sociedade. 


\section{REFERÊNCIAS}

1. Brasil. Ministério da Saúde. Intoxicação por Remédios: os perigos dentro de casa. Brasília: Ministério da Saúde; 2009.

2. Pereira AS, Lira SVG, Xavier EP, Vieira LJES. Produção sobre acidentes e violência apresentada em encontros de iniciação científica. Rev Enferm UERJ. 2007;15(2):218-22.

3. Ciampo LAD, Rubens GR, Gerson M. Acidentes domésticos na infância na área de Vila Lobato. Pediatria. 1997;19(1):38-42.

4. Brasil. Ministério da Integração Nacional. Redução das vulnerabilidades aos desastres e acidentes na infância. Secretaria Nacional de Defesa Civil. 2a ed. Brasília: Ministério da Integração Nacional; 2002.

5. Coelho LP, Rodrigues BMR. O cuidar da criança na perspectiva da bioética. Rev Enferm UERJ. 2009;17(2):188-93.

6. Waksman RD, Gikas RMC, Blank D. Prevenção de acidentes na infância e adolescência. São Paulo: Conselho Estadual dos Direitos da Criança e do Adolescente; 2009.

7. Wong DL. Enfermagem Pediátrica - elementos essenciais à intervenção efetiva. Rio de Janeiro: Guanabara Koogan; 2005.

8. Ciampo LAD, Ricco RG. Acidentes na infância. Pediatria. 1996;18(4):193-7.

9. Blank D. Prevenção e controle de injúrias físicas: saímos ou não do século 20? J Pediatria. 2002;78:84-6.

10. Brasil. Ministério da Saúde. Política Nacional de Redução da Morbimortalidade por Acidentes e Violências. Ministério da Saúde; 2001.

11. Anep. Associação Nacional de Empresas de Pesquisa. Critério de Classificação Econômica Brasil. São Paulo: Anep; 2009. 12. Amorim MGR, Medeiros GX, Benicio JA, Oliveira PSB, Santos EV, Sousa FF. Incidência e principais causas de acidentes domésticos em crianças na fase toddler e pré-escolar. Rev Coopex Fip Científca. 2009;1(1):1-7.

13. Ciampo LAD, Ferraz IS, Tazima MFGS, Bachette LG, Ishikawa K, Paixão R. Características clínicas e epidemiológicas de crianças acidentadas atendidas em um serviço de pronto-atendimento. Pediatria. 2011;33(1):29-34.

14. Amaral EMS, Silva CLM, Pereira ERR, Guamieri G, Brito GSS, Oliveira LM. Incidência de acidentes com crianças em um pronto-socorro infantil. Rev Inst Ciên Saúde. 2009;27(4):313-7.

15. Santos BZ, Grosseman S, Silva JYB, Cordeiro MMR, Bosco VL. Injúrias não intencionais na infância: estudo piloto com mães que frequentam a Clínica de Bebês da Universidade Federal de Santa Catarina, Brasil. Pesq Bras Odontoped Clin Integr. 2010;10(2):157-61.

16. Sociedade Brasileira de Pediatria. Aspiração de corpo estranho. São Paulo: Sociedade Brasileira de Pediatria; 2010.

17. Gámez HMC, Behar RR, García FR, Cordobés EV, Infante CS, Morales IA. Accidentes por cuerpos extraños en las vías respiratorias bajas en el niño. Rev Cubana Pediatr. 2009;81(3):1-11.

18. Lima RP, Barbosa-Ximenes L, Silva-Joventino E, Vieira LJES, Oriá, MOB. Acidentes na infância: local de ocorrência e condutas dos familiares no âmbito domiciliar. Enferm Global. 2009;15(2):1-13.

19. Vendrusculo TM, Balieiro CRB, Echevarría-Guanilo ME, Farina Jr JA, Rossi LA. Queimaduras em ambiente doméstico: características e circunstâncias do acidente. Rev Lat Am Enfermagem. 2010;18(3):444-51.

20. Sociedade Brasileira de Pediatria. Crianças e Adolescentes Seguros. Guia completo para prevenção de acidentes e violências. Sociedade Brasileira de Pediatria. São Paulo: Publifolha; 2005.

Recebido em: 10 de setembro de 2013. Versão atualizada em: 3 de outubro de 2013. Aprovado em: 24 de outubro de 2013. 